\title{
Anomalous Origin of the Left Coronary Artery from the Pulmonary Trunk: Late Diagnosis and Asymptomatic Evolution
}

\author{
Andrea Maria Giovannini Bercht, Marcos Danillo Peixoto Oliveira, Diego Simões Peniche, Sonia Maria F M Mesquita,
} Nana Miura

Instituto do Coração do Hospital das Clínicas da Faculdade de Medicina da Universidade de São Paulo, São Paulo, SP - Brazil

\section{Introduction}

Coronary artery anomalies (CAA) are congenital abnormalities in their origin, course and/or structure. Many controversies persist in terms of incidence, classification, tracking, heredity and treatment. Although they may be mostly asymptomatic, their clinical presentation in adults may result from myocardial ischemia, presenting angina pectoris, syncope, arrhythmias and sudden cardiac death (SCD). ${ }^{1-3}$

The origin of the left coronary artery (LCA) from the pulmonary trunk (PT), also known as the Bland-White-Garland syndrome, is a rare CAA, usually detected in childhood. It presents high mortality during the first years of life, but some patients may have their diagnosis only in adulthood. ${ }^{4}$ The clinical evolution is very unspecific and variable due to the diversity of anatomical abnormalities, associated or not with other structural malformations. As a result, some patients with this anomaly may remain asymptomatic for many years. ${ }^{5}$

\section{Case Report}

White woman, 67 years old, with history of systemic hypertension, hypercholesterolemia, hypothyroidism and type 2 diabetes mellitus, all under regular drug therapy (atorvastatin $20 \mathrm{mg} /$ day, ezetimibe $10 \mathrm{mg} /$ day, atenolol $50 \mathrm{mg} /$ day, enalapril 20 mg/day, levothyroxine 50 mcg/day, metformin 1700 mg/day). The patient denied previous episodes of myocardial infarction, stroke, coronary artery disease (CAD) or personal and family history of sudden cardiac death. Under outpatient follow-up in our service since 2009, the patient reported a poorly defined, unspecific clinical condition described as sporadic epigastric pain, of the burning type, worsening during meals, unrelated to exertion. Clinical examination did not reveal any significant abnormalities.

In another center, the patient underwent coronary angiography in 1999, which did not reveal any obstructive

\section{Keywords}

Heart Defects, Congenital/mortality; Coronary Vessel Anomalies/surgery; Pulmonary Artery/abnormalities; Diagnosis.

Mailing Address: Andrea Maria Giovannini Bercht •

Instituto do Coração do Hospital das Clínicas da Faculdade de Medicina da Universidade de São Paulo

Av. Dr. Enéas de Carvalho Aguiar, 44. Postal Code 05403-900,

Pinheiros, São Paulo, SP - Brazil

E-mail: deiadmg@uol.com.br

Manuscript received January 22, 2016; revised March 3, 2016; accepted April 19, 2016.

DOI: $10.5935 / 2318-8219.20160026$ lesions in the coronary arterial bed (data collected from medical records of the institution; images not available).

Complementary tests requested during the outpatient follow-up in our service: simple chest X-ray — with no significant abnormalities; electrocardiogram (ECG) at rest - sinus, $60 \mathrm{bpm}$, rare and isolated supraventricular ectopic beats, and nonspecific intraventricular conduction disturbance; myocardial perfusion scintigraphy (MIBI-adenosine) (2009) — mild transient low uptake in the left ventricular (LV) anterior wall (basal and middle segments); transthoracic two-dimensional echocardiogram (ECHO) at rest (2013) - global preserved RV and LV systolic function; LV ejection fraction $=0.56$; moderate mitral regurgitation; increased LV dimensions (63 x $44 \mathrm{~mm}$ ); mild eccentric myocardial hypertrophy; pulmonary artery systolic pressure $=38 \mathrm{mmHg}$; dilation of the right coronary artery (RCA) origin $(1.3 \mathrm{~cm})$; it was not possible to see the LCA origin; abnormal interventricular septal flow on Doppler.

Over regular six-monthly consultations, the patient denied recurrence of the above symptoms, as well as the emergence of other complaints.

Considering the ECHO findings, it was decided to conduct detailed anatomical study of computed tomography angiography of the coronary arteries (2015), which revealed: anomalous origin of the LCA from the PT; RCA with usual origin and path, providing an exuberant network of epicardial collateral arteries to the anterior descending artery; epicardial vessels noticeably dilated and tortuous; total calcium score of zero and absence of significant coronary luminal reductions (Figures 1 and 2).

Surgical correction of such congenital coronary anomaly was indicated according to the recommendations of national and international guidelines. Nevertheless, the patient and her family expressed refused the surgery. Therefore, the conservative strategy with regular outpatient follow-up was adopted for the case.

\section{Discussion}

CAA are congenital abnormalities in the origin, path and structure of the epicardial coronary arteries. ${ }^{1}$ Angelini ${ }^{6,7}$ described their incidence in the general population, of about $1 \%$. To date, there are no concrete data on different incidence rates for men and women or even between ethnicities or races. ${ }^{1}$

As a rule of thumb, the diagnosis of CAA is a challenge, as the patients are usually asymptomatic and their clinical examination do not often reveal specific findings. The same 


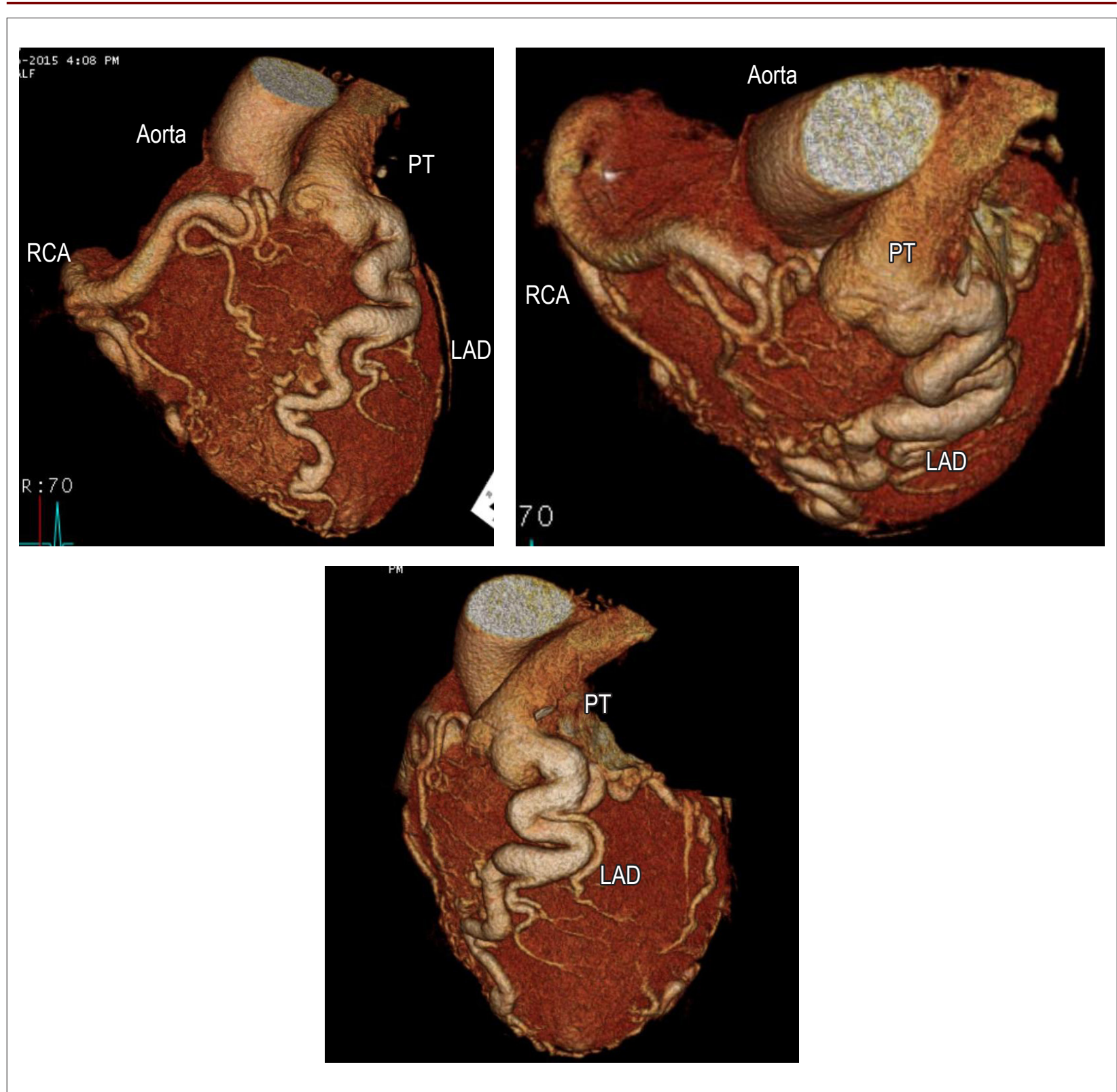

Figure 1 - Three-dimensional reconstruction (CT angiography) of the coronary circulation. Anomalous origin of the LCA from the PT. RCA with usual origin and route provides an exuberant network of epicardial collateral arteries to the anterior descending artery. Noticeable dilatations and tortuosity of the epicardial vessels. CT angiography: computed tomography angiography of the coronary arteries; LCA: left coronary artery; PT: pulmonary trunk; RCA: right coronary artery; LAD: left anterior descending.

applies to ECG at rest. Abnormalities suggesting ischemia and/or arrhythmia, especially in children or young adults, may raise suspicion and direct to other complementary diagnostic methods. Transthoracic ECHO presents better results in children than in adults, making it easier to identify the LCA. Conventional coronary angiography was traditionally considered the reference standard for the diagnosis of CAA. Nevertheless, the development of new imaging techniques, especially CT angiography and magnetic resonance imaging (MRI) of the heart, which allow a three-dimensional assessment of the origin, path and the relationship of the coronary arteries with adjacent structures, has shown some flaws of invasive coronary angiography in the diagnosis of CAA. ${ }^{1}$

The American Heart Association ${ }^{8}$ guidelines attribute class I indication with level of evidence $B$ to cardiac $C T$ angiography and MRI in the diagnosis of CAA. Once CT angiography is more widely available, this is the method of choice in most cases of suspected CAA. ${ }^{1}$

Surgical intervention is always indicated in the presence of anomalous origin of LCA from the PT, although it is suspected that in patients with this anomaly, prone to sudden death, surgery cannot prevent such 

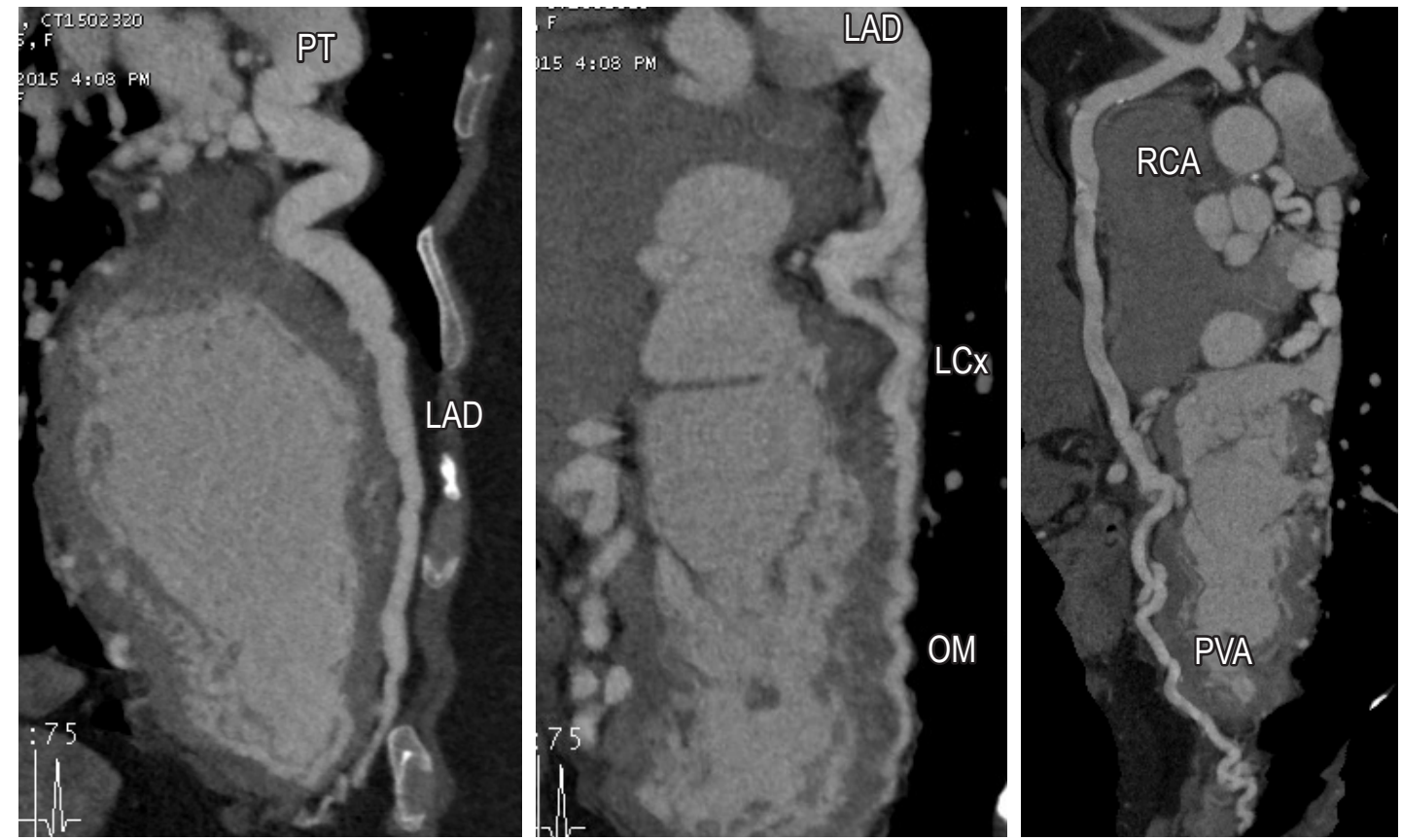

Figure 2 - Multiplanar reformatting (CT angiography) of the coronary circulation. CT angiography, computed tomography angiography of the coronary arteries. PT: pulmonary trunk; LAD: left anterior descending; LCx: left circunflex; RCA: right coronary artery; PVA: posterior ventricular artery; OM: obtuse marginal.

occurrence. ${ }^{4}$ Literature data ${ }^{9}$ show the effectiveness of LCA reimplantation in the aorta, or anastomosis between the left internal thoracic artery and the anterior descending coronary artery with LCA ligature.

The few patients who survive to adulthood may be asymptomatic if the collateral circulation is adequate, as in this case. However, the first symptom may be an acute myocardial infarction or SCD. ${ }^{4}$

Most patients (80\% to $85 \%$ ) without collateral circulation supply may progress to congestive heart failure secondary to myocardial ischemia with high mortality (about 90\%) without surgical treatment. ${ }^{10}$

In this case, immediately after diagnosis, surgical approach was indicated for the patient. However, that did not happen due to express refusal of the patient and her family. In these situations, a fairly frequent and regular follow-up is imperative. The emergence of left ventricular dysfunction and/or complex ventricular arrhythmias should reiterate the need for surgical intervention.

\section{References}

1. Almeida C, Dourado R, Machado C, Santos E, Pelicano N, Pacheco M, et al., Anomalias das artérias coronárias. Rev Port Cardiol. 2012;31(7-8):477-84.

2. Oliveira MD, de Melo PH, Esteves Filho A, Kajita LJ, Ribeiro EE, Lemos PA. Type 4 dual left anterior descending artery: a very rare coronary anomaly circulation. Case Rep Cardiol. ID 580543:1-3.

3. Oliveira MD, de Fazzio FR, Mariani J Jr, Campos CM, Kaita LJ, Ribeiro $E E$, et al. Superdominant right coronary artery with absence of left

\section{Authors' contributions}

Research creation and design: Bercht AMG, Oliveira MDP; Data acquisition: Bercht AMG, Oliveira MDP, Peniche DS; Data analysis and interpretation: Bercht AMG, Oliveira MDP, Mesquita SMFM, Miura N; Manuscript drafting: Bercht AMG, Oliveira MDP; Critical revision of the manuscript as for important intellectual content: Bercht AMG, Oliveira MDP, Peniche DS, Mesquita SMFM, Miura N.

\section{Potential Conflicts of Interest}

There are no relevant conflicts of interest.

\section{Sources of Funding}

This study had no external funding sources.

\section{Academic Association}

This study is not associated with any graduate program. circumflex and anomalous origin of the left anterior descending coronary from the right sinus: an unheard coronary anomaly circulation. Case Rep Cardiol. 2015;2015:721536.Epub 2015 Jul 09.

4. Jacob JLB, Salis FV. Anomalous origino $f$ the left coronary artery from the pulmonar trunk in a 45-year-old woman. Arq Bras Cardiol. 2003;81(1):196-8.

5. Pfeiffer MET. Anomalia congênita de coronária - relevância clínica, exercício e morte súbita. Rev DERC. 2013;19(4):114-8. 
6. Angelini P. Coronary artery anomalies-current clinical issues definitions, classification, incidence, clinical relevance, and treatment guidelines. Tex Heart Inst J. 2002;29(4):271-8.

7. Angelini P. Coronary artery anomalies: an entity in search of an identity. Circulation. 2007;115(10):1296-305.

8. Warnes CA, Williams RG, Bashore TM, Child JS, Connolly HM, Dearani JA, et al. ACC/AHA 2008 Guidelines for the management of adults with congenital heart disease: a report of the American College of Cardiology/American Heart Association Task Force on Practice Guidelines (Writing Committee to Develop Guidelines on the
Management of Adults With Congenital Heart Disease)," Circulation. 2008;118(23):e714-833

9. Oliveira SA, Diament J, Carvalho VB, Ariê S, Macruz R, Zerbini EJ. Anomalous origin of the left coronary artery from the pulmonary artery. Surgical repair of on unused form. J Cardiovasc Surg.(Torino). 1977;18(6):599-605.

10. Rodriguez-Gonzalez M, Tirado AM, Hosseinpour R, de Soto JS Anomalous origin of the left coronary artery from the pulmonary artery: diagnoses and surgical results in 12 pediatric patients. Tex Heart Inst J. 2015;42(4):350-6. 